\title{
CERTIFICAÇÃO LEED E SUA IMPORTÂNCIA NAS CONSTRUÇÕES BRASILEIRAS
}

\author{
LEED CERTIFICATION AND IT'S IMPORTANCE IN BRAZILIAN'S CONSTRUCTION
}

\author{
Alexandre da Silva Rech ${ }^{1}$ \\ Jean Debrassi ${ }^{2}$ \\ Leonardo Henrique Lira ${ }^{3}$ \\ Otávio Thomaz ${ }^{4}$ \\ Maicon Anderson de Souza ${ }^{5}$ \\ Recebido em: 31 jul. 2020 \\ Aceito em: 25 out. 2020
}

\section{RESUMO}

O desenvolvimento sustentável é um modelo de desenvolvimento capaz de garantir uma evolução durável que se sustente através dos tempos e com sucessão de gerações. Atualmente a sustentabilidade é um dos principais assuntos quando se trata da preocupação ambiental que a construção teve em todas suas etapas, tendo a certificação LEED como uma das principais bases para medir o desempenho ambiental de design, construção e manutenção de edifício buscando motivar e acelerar o desenvolvimento de práticas sustentáveis por meio da criação e implementação de critérios de desempenho e ferramentas universalmente entendidas e aceitas. Neste trabalho são relatados os fatores relacionados à certificação LEED e sua importância nas construções civis brasileiras através de revisão bibliográficas acerca do tema para seu embasamento. Portanto, torna-se fundamental o despertar da sociedade e de toda a cadeia produtiva do segmento da construção civil para repensar não só a metodologia LEED, mas também o comportamento e a responsabilidade de cada um na criação de espaços urbanos para se viver e trabalhar, realmente mais sustentáveis. Ao analisar esse estudo foi possível verificar que obter a certificação não é simples e são necessários uma série

\footnotetext{
${ }^{1}$ Acadêmico de Engenharia Civil (UNIFEBE).E-mail: xande_sc@unifebe.edu.br

${ }^{2}$ Acadêmico de Engenharia Civil (UNIFEBE).E-mail: jeandebrassi@hotmail.com

${ }^{3}$ Acadêmico de Engenharia Civil (UNIFEBE).E-mail: leonardohenriquelira@hotmail.com

${ }^{4}$ Acadêmico de Engenharia Civil (UNIFEBE).E-mail: otavio-thomaz@hotmail.com

${ }^{5}$ Docente do curso de Engenharia Civil (SINERGIA, CNEC e UNIASSELVI) e do curso de Arquitetura (UNIVALI). E-mail: eng_maicon@hotmail.com. Graduado em Engenharia Civil e Mestre em Engenharia de Produção (UNISOSIESC).
} 
de procedimentos para o mesmo além de despertar na sociedade e de toda a cadeia produtiva do segmento da construção civil para repensar não só a metodologia LEED, mas também o comportamento e a responsabilidade de cada um na criação de espaços urbanos para se viver e trabalhar, realmente mais sustentáveis.

PALAVRAS-CHAVE: Sustentabilidade, leed, construção civil.

\section{ABSTRACT}

Sustainable development is a model of development capable of ensuring a sustainable evolution that is sustained through time and with succession of generations. Sustainability is one of the main issues when it comes to the environmental concern that construction has had in all its stages, with LEED certification as one of the main bases for measuring the environmental performance of building design, construction and maintenance, seeking to motivate and accelerate the development of sustainable practices through the creation and implementation of universally understood and accepted performance criteria and tools. In this work, the factors related to LEED certification and its importance in Brazilian civil construction are reported through bibliographic review on the theme for its foundation. Therefore, it is fundamental to awaken society and the entire productive chain of the civil construction segment to rethink not only the LEED methodology, but also the behavior and responsibility of each one in the creation of urban spaces to live and work, really more sustainable. In analyzing this study it was possible to verify that obtaining the certification is not simple and a series of procedures are necessary for the same as well as awakening in society and the entire productive chain of the construction segment to rethink not only the LEED methodology, but also the behavior and the responsibility of each one in the creation of urban spaces to live and to work, really more sustainable.

KEYWORDS: Sustainability, leed, construction.

\section{INTRODUÇÃO}

Segundo a Commission on Environment and Development - WCED (1987), também conhecida como Comissão Brundthland, desenvolvimento sustentável seria o desenvolvimento que atende as necessidades presentes sem comprometer a habilidade das gerações futuras de atender suas próprias necessidades.

O termo "construção sustentável" foi proposto pela primeira vez em dezembro de 1993, pelo Prof. Charles Kibert da Universidade da Flórida, para 
apresentar as responsabilidades da indústria da construção civil no que diz respeito aos conceitos e objetivos da sustentabilidade, revelando uma necessidade premente para se atingir tais objetivos (PINTO; INÁCIO, s.d).

A avaliação do desempenho ambiental de edifícios surgiu com a constatação de que, mesmo os países que acreditavam dominar os conceitos de projeto ecológico, não possuíam meios para verificar o quão "verde" eram de fato os seus edifícios. A classificação de desempenho atrelada aos sistemas de certificação é um dos métodos mais eficientes para elevar o nível de desempenho ambiental tanto do estoque construído quanto de novas edificações (SILVA, 2003).

A certificação LEED foi elaborado e publicado, pelo United States Green Building Council (USGBC)6, na sua primeira versão, em 1999, com a finalidade de motivar e acelerar o desenvolvimento de práticas sustentáveis por meio da criação e implementação de critérios de desempenho e ferramentas universalmente entendidas e aceitas (USGBC, 2015).

É um método de classificação baseado na harmonização, ponderação de créditos (em função do impacto ambiental e da saúde humana) e regio-nalização. A eficiência energética e redução da emissão de CO2 são itens considerados de maior importância neste sistema de avaliação. O selo certifica edifícios a partir de uma lista de pré-requisitos e créditos, e possui quatro níveis: Certificado, Prata, Ouro e Platina (USGBC, 2015). O confronto entre desenvolvimento econômico e meio ambiente vem à tona a todo o momento, como no polêmico tema da assunção de metas para a redução de emissões poluentes por países em desenvolvimento ou países desenvolvidos e grandes emissores. Qualquer que seja o assunto fica no ar a interrogação se a humanidade será capaz de arcar no futuro com as consequências das suas atitudes contemporâneas (PARDINI, 2009).

Segundo Faria (2014), diretor do Green Building Council Brasil, cada vez mais engajado nos movimentos relacionados à sustentabilidade, o Brasil conquistou mais uma marca histórica. No último relatório da USGBC publicado em 2015, o Brasil ocupa o quinto lugar no ranking mundial em empreendimentos que buscam a certificação LEED (Leadership in Energy and Environmental Design). Para

\footnotetext{
${ }^{6}$ USGBC é uma organização sem fins lucrativos que tem por finalidade a divulgação de práticas sustentáveis em edifícios. É formada por mais de 12.000 organizações da indústria de construção, incluindo, entre outros, proprietários e usuários finais de edifícios. (USGBC, 2015).
} 
se ter uma ideia do quão representativo são esses números, o selo internacional LEED está presente em 153 países, e é considerado a principal certificação em sustentabilidade no mundo. (USGBC, 2015)

O presente artigo tem como objetivo uma revisão bibliográfica acerca do tema certificação LEED e sua importância nas construções civis brasileiras. Será abordado também seu contexto civil e ambiental, desafios para sua implantação e seus custos e benefícios.

\section{MATERIAIS E MÉTODOS}

O método de pesquisa consistiu no ponto de vista descritivo, pautado na revisão bibliográfica.

Para Marconi e Lakatos (2003), a pesquisa bibliográfica, ou de fontes secundárias, abrange toda bibliografia já tornada pública em relação ao tema de estudo, desde publicações avulsas, boletins, jornais, revistas, livros, pesquisas, monografias, teses, material cartográfico etc. Sua finalidade é colocar o pesquisador em contato direto com tudo o que foi escrito, dito ou filmado sobre determinado assunto.

Para Manzo (1971, p. 32), a bibliografia pertinente, "oferece meios para definir, resolver, não somente problemas já conhecidos, como também explorar novas áreas onde os problemas não se cristalizaram suficientemente".

\section{DESENVOLVIMENTO}

Muito se tem veiculado na mídia sobre edificações sustentáveis, construções sustentáveis e greenbuildings, nomes dados ao mesmo produto porém com semânticas diferentes. A expressão "greenbuilding", como originalmente criada, referia-se exclusivamente à dimensão ambiental da sustentabilidade. Porém, com o tempo acabou por identificar as edificações no contexto de sustentabilidade. A expressão "construções sustentáveis" foi cunhada para englobar todas as iniciativas dedicadas à criação de construções que utilizem recursos de maneira eficiente e que tenham maior longevidade (SILVA, 2003).

O desenvolvimento das metodologias de avaliação ambiental de edifícios 
que surgiram na década de 90 na Europa, EUA e Canadá foi embasado no conceito de análise do ciclo de vida (SILVA, 2003). Atualmente, quase todos os países europeus, além dos EUA, Canadá, Austrália, Japão e Hong Kong, possuem um sistema de avaliação ambiental de edifícios (SILVA; AGOPYAN, 2004).

Os mesmos autores acima ressaltam que - com exceção do SBAT, método sul-africano - todos os métodos de avaliação de edifícios hoje existentes focam exclusivamente na dimensão ambiental, uma vez que estão baseados em modelos elaborados por países desenvolvidos. Neles, a sociedade já encontrou equilíbrio de qualidade de vida, igualdade social e distribuição de renda e a prioridade passa a ser o restabelecimento dos recursos naturais destruídos.

O LEED é um sistema único que é aplicado em todo o mundo. Não existe um LEED diferente para o Brasil e para a China, por exemplo. Em vez de criar versões regionais do sistema de classificação, o mercado usa o mesmo sistema em todo o mundo. Como forma de proporcionar o intercâmbio e o desenvolvimento técnico entre os diferentes países, foi criado o LEED International Roundtable. Formado por um grupo de 38 organizações, a maioria dos quais representam um país onde está presente e atuante através de seus Green Building Councils locais. As discussões giram em torno do impacto e aplicação do sistema por todos os países. Cada um de seus componentes serve como um grupo de auxílio do USGBC para o fomento e adaptabilidade da Certificação LEED localmente, com discussões de nível internacional.

O LEED e a sua equipe, através do USGBC, funcionam como a inteligência central que une os conceitos, mas a função das mesas redondas LEED é distribuir essa inteligência para todos os outros países. Cada um deles conhece a indústria da construção regional, local e as práticas e normas que melhor funcionam onde eles estão contribuindo para melhorar a aplicabilidade das normas (Revista GBC Brasil, 2015).

O LEED trabalha nos níveis global, regional e local de forma integrada. LEED é global, pois é o mesmo sistema normatizado e padronizado que é utilizado em todas as partes do mundo. Isso significa que qualquer pessoa pode comparar um edifício em São Paulo com um em Nova York ou Xangai ou Estocolmo, com muita clareza (Scot Horst, membro do USGBC). 
HISTÓRICO DO LEED NO BRASIL

Segundo Faria (2014), diretor do Green BuildingCouncil Brasil, cada vez mais engajado nos movimentos relacionados à sustentabilidade, o Brasil conquistou mais uma marca histórica. Um dos fatores de destaque do país neste ranking é atribuído a presença de profissionais altamente qualificados atuantes na indústria nacional de greenbuilding. Outro detalhe importante é a conscientização e conhecimento dos benefícios atrelados às práticas de construção sustentável.

Em 2013, a média de empreendimentos certificados foi equivalente a 4,5 projetos por mês. Já em 2014, considerando apenas o primeiro semestre, esse valor chegou a sete projetos certificados por mês. Comparando os primeiros semestres de 2013 e 2014, foram 27 empreendimentos certificados no primeiro ano e 42 no segundo. O crescimento comparativo é de 55,55\% (FARIA, 2014).

Entre as edificações certificadas em 2014, se destacam: o Estádio do Mineirão, um dos únicos estádios no mundo a conseguir a certificação LEED NC Platinum; o Museu de Artes do Rio de Janeiro, LEED NC Silver; o bairro planejado Ilha Pura, que será a Vila dos Atletas, durante as Olimpíadas de 2016, 1 o empreendimento LEED ND (Neighborhood) da América Latina; Colégio Positivo Internacional de Curitiba certificado pelo LEED School Gold; a nova fábrica da GM em Joinville, LEED Gold; e a sede da AMCHAM certificada LEED EBOM Gold. São diferentes tipologias de edificações e "rating system" demonstrando como o movimento se expande a diversos setores da construção civil, não ficando restrito as edificações corporativas. Hoje somos o segundo país com o maior número de edificações esportivas certificadas LEED e o segundo país com o maior número de projetos LEED ND registrados. (FARIA, 2014).

\section{DESENVOLVIMENTO SUSTENTÁVEL E A CONSTRUÇÃO CIVIL}

O desenvolvimento sustentável é um modelo de desenvolvimento capaz de garantir uma evolução que dure, que se sustente através dos tempos e com sucessão de gerações (HERNANDES, 2006).

A definição clássica de desenvolvimento sustentável foi elaborada pela World Commissionon Environmentand Development - WCED, no ano de 1987, também conhecida como Comissão Brundthland: "desenvolvimento sustentável é 
o desenvolvimento que atende as necessidades presentes sem comprometer a habilidade das gerações futuras de atender suas próprias necessidades" (WCED, 1987).

O conceito de desenvolvimento sustentável está apoiado em três pilares: o social, pedindo uma sociedade mais justa em relação ao desenvolvimento humano e qualidade de vida; o ambiental, solicitando equilíbrio entre a proteção e o consumo dos recursos naturais; e o econômico requerendo acesso aos recursos e oportunidades sem ferir os limites ecológicos e os direitos humanos. Contrário ao que se imagina, o desenvolvimento sustentável não é um objetivo, mas um tipo de desenvolvimento necessário para atingir um estado de sustentabilidade (AGENDA 21 CSPD, 2002).

Quando se observa as cidades com suas casas, edifícios, indústrias, escolas, hospitais, bem como toda a infra-estrutura, fica evidente a importância das atividades relacionadas à construção civil para um país. Também são visíveis os impactos por ela gerados, alguns de caráter transitório como alterações no trânsito, poeira e barulho, outros permanentes, como a permeabilidade do solo, mudando o regime de drenagem, causando enchentes e reduzindo as reservas de água subterrânea (CBCS, 2007).

Os números da construção civil no Brasil são expressivos. A Pesquisa Anual da Indústria da Construção - PAIC de 2006 mostra o importante papel da indústria da construção civil na economia, onde as empresas pertencentes a este segmento foram responsáveis, naquele ano, por obras e serviços no valor de $\mathrm{R} \$ 110,7$ bilhões (VASCONCELLOS, 2006).

\section{RESULTADOS E DISCUSSÕES}

A seguir serão discutidos os resultados e discussões do presente artigo, tendo como base a importância do LEED no contexto civil e ambiental, desafios para sua implantação e seus custos e benefícios.

IMPORTÂNCIA DO LEED NO CONTEXTO CIVIL E AMBIENTAL

Pode-se dizer que metodologias como o LEED trouxeram à tona discussões sobre questões ambientais relacionadas à Indústria da Construção Civil 
anteriormente jamais pensadas, tais como: Impactos causados pela Construção Civil, não só na fase de produção, mas também no período de operação de uma edificação, abrindo assim a discussão sobre as responsabilidades e o papel deste segmento na preservação do planeta, de forma a se procurar por sistemas construtivos, tecnologias e processos menos agressivos tanto no uso de recursos naturais como a água, energia e matéria-prima, quanto na emissão de poluentes e resíduos (SILVA, 2009)

A obtenção da certificação LEED promove o envolvimento de toda a cadeia produtiva no processo, fazendo com que haja a disseminação de conhecimento. Projetistas, fornecedores de insumos e mão de obra e até mesmo os clientes finais são forçados a adquirir conhecimento, não somente do processo de certificação, mas também de questões mais amplas relacionadas a ele. (SILVA, 2009)

\section{DESAFIOS PARA A IMPLANTAÇÃO}

Sendo o LEED uma metodologia que visa a melhorar o desempenho ambiental das edificações e que a atribuição de certificação representa para o mercado a constatação de que as edificações certificadas são melhores e prejudicam menos o meio ambiente, logo, a conclusão sobre seu uso é de que as empresas que a exigem têm uma preocupação maior com o meio ambiente. A lógica exposta é passível de questionamentos. A certificação demonstra uma real preocupação em relação ao meio ambiente? Será que as edificações com certificado LEED são mais sustentáveis? Qual a base comparativa para que estas edificações sejam consideradas melhores em relação ao seu desempenho ambiental?

O uso generalizado em todos os países, sem alguma distinção, faz com que não levemos em consideração as agendas particulares de cada um deles, discutindo no mesmo nível problemas com gravidades distintas em países desenvolvidos e em desenvolvimento. A metodologia LEED foi desenvolvida dentro dos EUA, país industrializado, onde, superadas as preocupações com problemas básicos sociais, como suprimento de água e rede de esgoto para toda a população, voltam-se às questões ambientais, como explicitado na categoria Energia e Atmosfera (SILVA, 2009).

Além disso, Hernandes e Duarte (2007) fizeram em seu artigo uma análise 
das alterações da versão atual do LEED v.2.2, comparando-as com a versão imediatamente anterior v.2.1 e concluíram que das 26 alterações feitas, 13 delas diminuíam o grau de exigência na busca dos seus requisitos. Isto levanta a dúvida referente ao papel desta metodologia de liderar o mercado rumo a níveis mais elevados de desempenho ambiental, uma vez que, aparentemente, o sistema está se adaptando ao mercado e não o inverso.

Para Silva (2003), o LEED bem como a maioria das metodologias existentes, avalia exclusivamente a dimensão ambiental, deixando de lado as questões sociais, tão importantes para países em desenvolvimento, como o Brasil. Para se dar o nome de edificação sustentável a um edifício, torna-se imprescindível a inclusão da dimensão social na sua avaliação.

É certo que o conhecimento e entendimento detalhado da estrutura da metodologia LEED, bem como de suas alterações ao longo dos anos traz dúvidas sobre práticas típicas e simples nos EUA e que se tornam insanas quando trazidas para a realidade brasileira, com grandes custos ambientais e por vezes econômicos e financeiros, como por exemplo a falta de fornecedores e o uso de madeira maciça em vez de laminada ou aglomerada; relacionadas à sua capacidade de cumprir o seu objetivo mais relevante de ter metas ambientais mais desafiadoras para projetos de edificações e quanto ao seu uso indiscriminado em todos os países, sem a preocupação com as agendas de cada um deles, principalmente quando existem problemas sociais mais graves e prioritários a serem resolvidos, como é o caso do Brasil. Por outro lado, também é correto afirmar que todos estes questionamentos decorrentes da introdução de uma metodologia de desempenho ambiental são apenas a ponta visível do iceberg (SILVA, 2009).

\section{CUSTOS E BENEFÍCIOS}

Satisfazer e atender às expectativas geradas por diferentes clientes não é tarefa fácil ainda mais quando o produto vendido é uma edificação. As particularidades do produto edifício provocam no comprador um comportamento complexo, uma vez que estão comprando um produto caro, com características únicas e geralmente considerado de longa duração. Neste contexto, entender os ganhos que as edificações sustentáveis podem trazer ou fazer com que os clientes/ usuários finais compreendam seus benefícios não é tarefa fácil, pois requer a 
demonstração do valor do produto e a ligação deste com os requisitos do cliente. $\mathrm{O}$ conceito de valor se refere à relação entre cliente e produto e o preço pago em um produto é, em última instância, a expressão do reconhecimento, pelo cliente, do valor daquele produto (MIRON; FORMOSO, 2003).

A teoria econômica neoclássica sugere que a alocação de recursos é determinada pela força do mercado. Se um recurso se torna escasso, seu preço sobe (lei da oferta-demanda) e, desta forma, é criada oportunidade de produção de produtos substitutos aos recursos faltantes (MYERS, 2004). Além disso, também de acordo com esta teoria, os indivíduos responsáveis pelas decisões selecionam uma determinada alternativa tendo por base a racionalidade, considerando suas preferências e sabendo das consequências de cada opção bem como dos seus respectivos riscos e incertezas (GLUCH; BAUMANN, 2004).

Os textos econômicos frequentemente enfatizam a dificuldade de se ter um produto e/ou serviço diferenciado na indústria da construção civil, seja pela qualidade ou pela confiabilidade, tendo em vista que existe uma grande quantidade de empresas que fazem produtos muito similares. A tendência da construção sustentável está emergindo lentamente e conquistando construtores e clientes (MYERS, 2004). O valor de um empreendimento tem sido julgado pela sua localização, qualidade, função e estética e os atributos de um edifício sustentável quase sempre são invisíveis e apreciáveis apenas depois da sua ocupação, durante a fase de uso. No entanto, está sendo criado um elo entre valor de mercado de edifícios e características sustentáveis e seu respectivo desempenho (DAVIES, 2005).

O valor dado a um empreendimento pode ter vários pontos de vista e dependerá do tipo de cliente, da contribuição deste empreendimento para a lucratividade do negócio principal e de razões subjetivas, com imagem, identidade e outras preferências pessoais (LÜTZKENDORF; LORENZ, 2005).

Para Handler (1970), os edifícios sustentáveis, além de contribuírem para o meio ambiente e apresentarem custos operacionais e de manutenção inferiores aos edifícios tradicionais, eles ainda desempenham papéis sociais junto à comunidade, seja pelo seu aspecto cultural, de saúde, social e cultural e junto à região e/ou município no qual ele está inserido, sendo o agente promotor da valorização da área e da geração de empregos diretos e indiretos. 
Davies (2005) aponta uma grande lacuna existente, de entendimento e conhecimento, entre a indústria sustentável e as entidades (pessoas ou organizações) responsáveis pela viabilidade de um empreendimento. De acordo com Ries e Bilec (2006), muitas áreas chave de negócio, como investidores e incorporadores, estão ansiosas por um entendimento estrutural e monetário de greenbuildings, bem como de sua métrica.

É preciso que se encontre uma maneira de quantificar melhor o valor agregado às construções sustentáveis na tentativa de explicitar claramente seus ganhos e o que as distingue, efetivamente, das construções tradicionais. O valor ambiental é o inverso do resultado da soma dos impactos causados durante o ciclo de vida de um edifício (BOGENSTÄTTER, 2000).

\section{CONCLUSÃO}

Se por um lado é de extrema importância a contabilização dos ganhos de sustentabilidade em um projeto, por outro, é imprescindível a educação daquelas pessoas que sentirão e usufruirão, ao longo do tempo, dos benefícios destas construções. Se a indústria conseguir convencer os consumidores usuários dos ganhos de uma construção sustentável, estas se tornarão mais desejadas e, provocará uma demanda do mercado.

Se a percepção do mercado é de que empreendimentos sustentáveis possuem custo inicial maior que os empreendimentos convencionais e é certa a afirmação de que os benefícios gerados por estes empreendimentos podem ser mais bem percebidos ao longo do seu ciclo de vida, vale analisar a viabilidade econômica de um empreendimento sob a ótica da sua vida útil. Neste caso, serão levados em conta alternativas de projeto, soluções tecnológicas e sistemas prediais, os quais definirão e caracterizarão este empreendimento, tendo por finalidade a redução do consumo de recursos naturais como água e energia, a diminuição da quantidade de resíduos sólidos e poluentes atmosféricos e, ao mesmo tempo, de proporcionar um ambiente mais saudável aos usuários finais da edificação.

Cabe também ressaltar que os empreendimentos sustentáveis trazem consigo oportunidades de redução de riscos empresariais, uma vez que existe uma grande cobrança da sociedade e mídia a respeito de questões voltadas para a 
sustentabilidade.

Portanto, torna-se fundamental o despertar da sociedade e de toda a cadeia produtiva do segmento da construção civil para repensar não só a metodologia LEED, mas também o comportamento e a responsabilidade de cada um na criação de espaços urbanos para se viver e trabalhar, realmente mais sustentáveis.

\section{REFERÊNCIAS}

Agenda 21 para Construção Sustentável em Países em Desenvolvimento. THEINTERNATIONALCOUNCIL FOR RESEARCH AND INNOVATION IN BUILDING AND CONSTRUCTION AND UNITED NATIONS ENVIORNMENT PROGRAM. África do Sul, 2002. $82 \mathrm{p}$.

Brasil: Saltando de avaliação ambiental para avaliação de sustentabilidade. São Paulo: Escola politécnica da USP - Departamento de Engenharia de Construção Civil, 2004. 12 p. BoletimTécnico - BT/PCC/376.

BOGENSTÄTTER, ULRICH. Prediction and optimization of life-cycle costs in earlydesign. Building Research \& Information, London, v. 28, p. 376-386, 2000.

DAVIES, ROSS. Green Value, Green Building, growing assets, Report 2005.Emhttp://www.rics.org/NR/rdonlyres/93B20864-E89E-4641-AB11028387737058/0/GreenValueReport.pdf, acesso em setembro de 2016.

FARIAS, F. Uma Marca Histórica. Revista GBC Brasil, São Paulo, n.1, p. 6, 2014.

HANDLER, BENJAMIN, A. Systems Approach to Architecture New York EUA:American Elsevier Publishing Company, Inc, 1970, 183 p.

HERNANDES, THIAGO; DUARTE, DENISE. LEED-NC como sistema de avaliação da sustentabilidade: questionamentos para uma aplicação local. Brasil - Campo Grande,MS. 2007. p. 442-451. ENCONTRO LATINO-AMERICANO SOBRE EDIFICAÇÕES ECOMUNIDADES SUSTENTÁVEIS, 2., 2007, Campo Grande, MS.

MARCONI, Marina; LAKATOS, Eva. Fundamentos da Metodologia de Pesquisa. São Paulo: Atlas S.A. 2003, 5o ed. 309 p.

MIRON, Luciana Ines Gomes; FORMOSO, Carlos Torres. Client requirementmanagement in building projects. Estados Unidos - Blacksburg. 2003. 
13 p. CONFERENCE OF THE INTERNATIONAL GROUP FOR LEAN CONSTRUCTION,11., Blacksburg, USA.

PARDINI, Andréa Fonsceca. Contribuição ao Entendimento da Aplicação da CertificaçaoLeed e do Conceito de Custos no Ciclo de Vida em Empreendimentos Mais Sustentáveis no Brasil. Dissertação apresentada à Comissão de Pós graduação da Faculdade de Engenharia Civil, Arquitetura e Urbanismo da Universidade Estadual de Campinas, 2009.

PINTO, A. REAES; INÁCIO, MARQUES M. A Evolução da Construção Civil no Sentido da Sustentabilidade. Contribuição para uma Estratégia Nacional. Lisboa, S.D.

SILVA, VANESSA GOMES. Avaliação da sustentabilidade de edifícios de escritórios brasileiros: diretrizes e base metodológica São Paulo, 2003. $210 \mathrm{f}$. Tese Departamento de Engenharia de Construção Civil, Escola politécnica da USP.

SILVA, VANESSA GOMES DA; AGOPYAN, VAHAN. Avaliação de edifícios no Brasil: Saltando de avaliação ambiental para avaliação de sustentabilidade. São Paulo: Escola politécnica da USP - Departamento de Engenharia de Construção Civil, 2004. 12 p. BoletimTécnico - BT/PCC/376.

UNITED STATES GREEN BUILDING COUNCIL. LEED Green Building RatingSystem For New Construction \& Major Renovations Version 2.2 - For Public Use and Display. Disponivel em: <www.usgbc.org>. Acesso em: 25 ago. 2016.

VASCONCELLOS, FRANCISCO. Construção: desempenho e perspectivas. Apresentação feita em 26 de julho de 2006 no Sindicel.

WORLD COMMISSION ON ENVIORNMENT AND DEVELOPMENT. Our Common Future. Londres: Oxford University Press, 1987. 400 p. 